\title{
What perturbs isotherms? An assessment using fission track thermochronology and thermal modelling along the Gotthard transect, Central Alps
}

\author{
C. Glotzbach1, C. Spiegel1,2, J. Reinecker1, M. Rahn3 and W. Frisch1 \\ 1 Institute of Geoscience, University Tübingen, Sigwartstr. 10, 72076 Tuebingen, Germany \\ (e-mail: christoph.glotzbach@uni-tuebingen.de) \\ 2 Now at FB 5 Geoscience, University Bremen, Postfach 33044028334 Bremen, Germany \\ 3 Institute of Mineralogy-Geochemistry, University Freiburg, Albertstr. 23b, 79104 Freiburg, Germany
}

Apatite composition (weight- $\%$ ) of 15 samples from the Gotthard road tunnel and corresponding surface transect and from Durango, with averages and standard deviations calculated from totally 320 electron microprobe analyses

\begin{tabular}{lcccccccccc}
\hline Sample & $\mathbf{n}$ & $\mathbf{F}$ & $\mathbf{C l}$ & $\mathbf{S i O}_{2}$ & $\mathbf{M n O}$ & $\mathbf{C e}_{2} \mathbf{O}_{3}$ & SrO & $\mathbf{P}_{2} \mathbf{O}_{5}$ & $\mathbf{C a O}$ & $\mathbf{D p a r}$ \\
\hline MRP 229 & 10 & $3.23 \pm 0.19$ & $0.100 \pm 0.020$ & $0.030 \pm 0.010$ & na & $0.04 \pm 0.02$ & bd & $44.00 \pm 0.61$ & $54.68 \pm 0.37$ & $1.42 \pm 0.09$ \\
MRP 231 & 49 & $3.70 \pm 0.10$ & bd & $0.012 \pm 0.020$ & $0.054 \pm 0.014$ & na & $0.036 \pm 0.043$ & $43.90 \pm 0.50$ & $54.90 \pm 0.70$ & $1.26 \pm 0.11$ \\
MRP 232 & 49 & $3.45 \pm 0.19$ & $0.013 \pm 0.006$ & $0.023 \pm 0.044$ & $0.103 \pm 0.039$ & na & $0.040 \pm 0.028$ & $43.80 \pm 0.40$ & $54.80 \pm 0.30$ & $1.34 \pm 0.13$ \\
MRP 233 & 9 & $2.72 \pm 0.19$ & $0.028 \pm 0.004$ & $0.010 \pm 0.010$ & na & $0.04 \pm 0.01$ & $0.040 \pm 0.020$ & $40.81 \pm 0.56$ & $54.65 \pm 0.32$ & $1.57 \pm 0.15$ \\
MRP 234 & 10 & $3.88 \pm 0.22$ & bd & $0.120 \pm 0.090$ & na & $0.11 \pm 0.05$ & bd & $43.69 \pm 0.77$ & $54.15 \pm 0.57$ & $1.41 \pm 0.10$ \\
MRP 236 & 10 & $4.02 \pm 0.18$ & bd & $0.150 \pm 0.100$ & na & $0.13 \pm 0.05$ & bd & $41.11 \pm 1.00$ & $54.12 \pm 0.60$ & $1.29 \pm 0.14$ \\
MRP 239 & 10 & $4.03 \pm 0.23$ & bd & $0.040 \pm 0.050$ & na & $0.06 \pm 0.02$ & bd & $44.46 \pm 0.50$ & $54.58 \pm 0.28$ & $1.25 \pm 0.10$ \\
MRP 241 & 7 & $3.48 \pm 0.08$ & bd & $0.030 \pm 0.020$ & na & $0.02 \pm 0.02$ & $0.020 \pm 0.020$ & $43.62 \pm 0.40$ & $54.71 \pm 0.10$ & $1.47 \pm 0.09$ \\
MRP 242 & 10 & $3.62 \pm 0.19$ & bd & $0.040 \pm 0.010$ & na & $0.05 \pm 0.03$ & $0.020 \pm 0.020$ & $42.40 \pm 0.24$ & $54.87 \pm 0.25$ & $1.41 \pm 0.09$ \\
MRP 244 & 49 & $3.47 \pm 0.19$ & bd & $0.057 \pm 0.071$ & $0.071 \pm 0.046$ & na & $0.100 \pm 0.065$ & $43.60 \pm 0.40$ & $54.90 \pm 0.40$ & $1.32 \pm 0.14$ \\
MRP 245 & 5 & $3.27 \pm 0.10$ & bd & $0.250 \pm 0.120$ & na & $0.19 \pm 0.09$ & $0.080 \pm 0.030$ & $42.85 \pm 0.78$ & $54.43 \pm 0.42$ & $1.63 \pm 0.10$ \\
MRP 247 & 12 & $4.09 \pm 0.11$ & bd & $0.210 \pm 0.080$ & na & $0.16 \pm 0.08$ & $0.020 \pm 0.010$ & $44.58 \pm 0.48$ & $54.01 \pm 0.35$ & $1.13 \pm 0.14$ \\
MRP 248 & 10 & $4.15 \pm 0.23$ & bd & $0.160 \pm 0.140$ & na & $0.14 \pm 0.04$ & bd & $43.99 \pm 0.36$ & $54.04 \pm 0.25$ & $1.12 \pm 0.12$ \\
MRP 249 & 10 & $3.75 \pm 0.22$ & $0.006 \pm 0.005$ & $0.150 \pm 0.040$ & na & $0.21 \pm 0.05$ & $0.020 \pm 0.020$ & $43.65 \pm 0.77$ & $54.48 \pm 0.26$ & $1.34 \pm 0.12$ \\
MRP 291 & 50 & $4.03 \pm 0.22$ & bd & $0.025 \pm 0.098$ & $0.520 \pm 0.290$ & na & $0.012 \pm 0.016$ & $43.40 \pm 0.40$ & $54.10 \pm 0.60$ & $1.26 \pm 0.12$ \\
Durango & 20 & $3.61 \pm 0.09$ & $0.410 \pm 0.020$ & $0.480 \pm 0.050$ & $0.010 \pm 0.010$ & $0.71 \pm 0.04$ & $0.050 \pm 0.020$ & $42.78 \pm 0.75$ & $53.46 \pm 0.33$ & $1.63 \pm 0.11$ \\
\hline
\end{tabular}

$\mathrm{n}$ is the number of measurements; na means not measured; bd means below detection, with detection limits in ppm:

F 140, Cl 40, SiO2 100, Ce2O3 180, $\mathrm{SrO} 170, \mathrm{P} 2 \mathrm{O} 5$ 470, CaO 105 
Shaded relief overview of the 3D finite difference model of the Gotthard transect with coordinates of the Swiss reference system. Different colours refer to the involved geological units: Aar massif, Gotthard massif, Tavetsch massif, Urseren zone and Schistes lustrées.

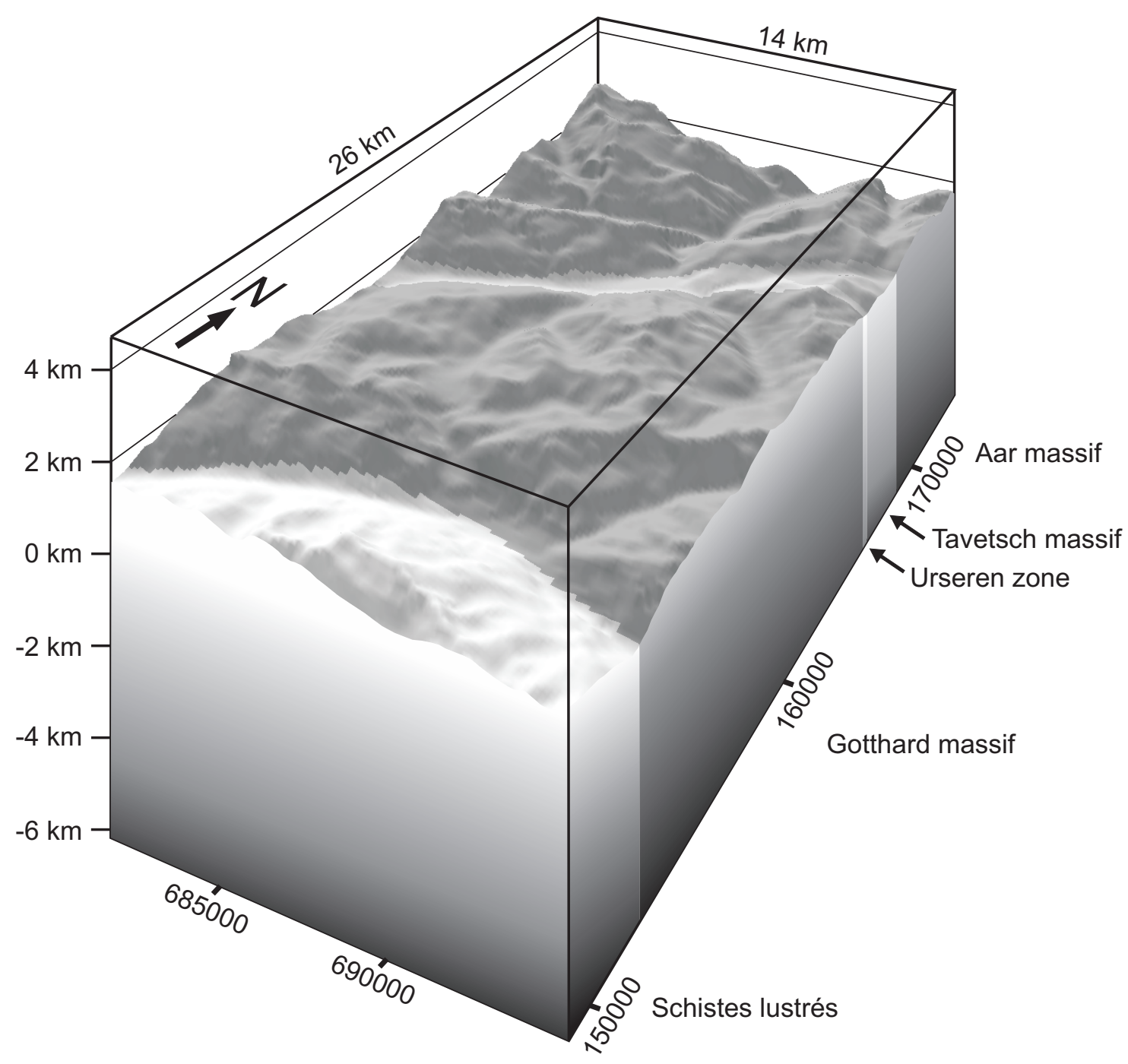


Geometrical, physical and thermal parameters used for numerical modelling

\begin{tabular}{llrr}
\hline Parameter & Description & Value & Unit \\
\hline $\mathrm{T}$ & temperature & variable & ${ }^{\circ} \mathrm{C}$ \\
$\mathrm{t}$ & time & variable & $\mathrm{S}$ \\
$\mathrm{x}, \mathrm{y}, \mathrm{z}$ & Cartesian coordinates & variable & $\mathrm{m}$ \\
$\Delta \mathrm{x}, \Delta \mathrm{y}, \Delta \mathrm{z}$ & nodes spacing & 132.9 & $\mathrm{~m}$ \\
$\mathrm{~S}$ & heat production & see Table 2 & $\mathrm{~W} \mathrm{~kg}^{-1}$ \\
$\mathrm{u}$ & exhumation rate & 0.45 & $\mathrm{~km} \mathrm{Ma}^{-1}$ \\
$\rho$ & density & 2700 & $\mathrm{~kg} \mathrm{~m}^{-3}$ \\
$\mathrm{c}_{\mathrm{p}}$ & specific heat & 1000 & $\mathrm{~J} \mathrm{~kg}^{-1} \mathrm{~K}^{-1}$ \\
$\mathrm{k}$ & thermal conductivity & see Table 2 & $\mathrm{~W} \mathrm{~K}^{-1} \mathrm{~m}^{-1}$ \\
$\mathrm{~T}_{0}$ & air T at sea level & 11.88 & ${ }^{\circ} \mathrm{C}$ \\
$\alpha$ & atmospheric lapse rate & 4.6 & ${ }^{\circ} \mathrm{C} \mathrm{km}^{-1}$ \\
$\mathrm{G}_{\mathrm{B}}$ & vertical bottom geothermal gradient & 20 & ${ }^{\circ} \mathrm{C} \mathrm{km}^{-1}$ \\
\hline
\end{tabular}

